\title{
Application of RFID technology in the vehicle alarm system
}

\author{
Zhang Jiangzhou ${ }^{1, a}$, Wang Shuai ${ }^{2, b}$ and Gao Ning ${ }^{3, c}$ \\ ${ }^{1}$ Shandong Jinan 540012 China \\ ${ }^{2}$ Shandong Jinan 540012 China \\ ${ }^{3}$ Shandong Jinan 540012 China \\ azhangjzh@sdas.org, ${ }^{b}$ wangsh@sdas.org, ${ }^{\mathrm{c}}$ gaoj@sdas.org
}

Keywords: RFID; Vehicle theft; Linkage alarm.

Abstract. Vehicle anti-theft alarm system using RFID technology in the region of the vehicle for security management and establish a vehicle intelligent anti-theft alarm and management platform, the vehicle to achieve the automatic import and export with owner identification, vehicle positioning, statistical statements, illegal mobile alarm, to solve the vehicle lost problem.

\section{Introduction}

Vehicle anti-theft problem has been is that the majority of owners and the police officer the thorny problems, in order to prevent stolen vehicles in the residential, office units and other public places, traditional security measures, such as the installation of expensive locks or assign the duty or import and export inventory etc. means, not only time-consuming laborious and the effect is not good, vehicle theft phenomenon is difficult to be effectively curbed.

RFID (radio frequency identification)technology, also known as radio frequency identification (RFID), is a kind of communication technology, through radio signals to identify a specific target and to read and write data without recognition system with specific target established between the mechanical or optical contact. RFID card, also known as the electronic tag or electronic bar code, in recent years, the rise of IOT technology is the core technology and one of the key components, is one of the countries are currently focus on the cultivation and promotion of new economic growth. RFID technology has the advantages of waterproof, antimagnetic, high temperature resistance, long life, far distance recognition, data encryption, not afraid of grease, anti dust pollution etc. advantages.

\section{System main advantages}

Vehicle anti-theft alarm system using RFID technology in the region of the vehicle for security management and establish a vehicle intelligent anti-theft alarm and management platform, the vehicle to achieve the automatic import and export with owner identification, vehicle positioning, statistical statements, illegal mobile alarm. Have the following technical advantages.

1) The advanced technology, networking technology and automatic sensing technology.

2) To take the initiative to prevent, the suspect vehicle is far away from the exit can be identified, has dispatched full time slot.

3) The signal reliability, good stability, strong anti-interference ability.

4) The safety is high, with 32 rolling code technology, effectively prevent the label be deciphered copy.

5) Is easy to use, no parking, in addition to the main vehicle without any operation, the legitimacy of automatic recognition.

6) The management is convenient, unattended, automatic alarm of illegal vehicles.

7) Is easy to deploy, only need to install the entrance door in the area.

8) The recognition rate is high, can achieve precise identification of more than $98 \%$, effectively prevent theft.

9) The complete function, providing a non motor vehicle management and charge management function. 


\section{Overall system design}

\section{System composition}

The vehicle anti-theft alarm system is divided into two blocks of hardware and software.. The hardware mainly includes: RFID card reader, RFID remote identification card, sound and light alarm, identification and alarm server etc.; software is mainly divided into: data acquisition and setting module, data extraction module, algorithm matching module, automatic alarm module.

System resource E-R diagram

The entity of the system is the user and the vehicle, the abstract to the logical relationship is the relationship between the user card and the vehicle card. User name can have a number of cars, a car head of a person can only be a person, and a car generally only a lock, and a lock can have more to open the key. Therefore, the logic relationship is: a car corresponding to a RFID card, and can be matched with the security through a number of RFID user card. Its E-R diagram is shown in Figure 1.

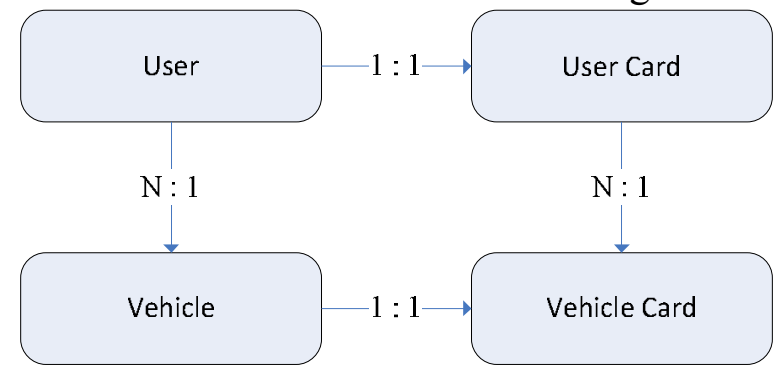

Figure 1 E-R diagram

System architecture design

The system of $\mathrm{B} / \mathrm{S} / \mathrm{D}$ distributed architecture design, data acquisition module, alarm module, detection service, database, etc.. Main functions of data acquisition is the collected data is uploaded to the service center through the LAN; alarm module is mainly according to the instruction inspection service and issued to the corresponding alarm terminal corresponding disposal; testing services for data collection and vehicle detection based on the detection algorithm, when there is an alarm, to alarm module to send commands. The database is storage system running the data acquisition, operation process data, disposal of the results, such as.

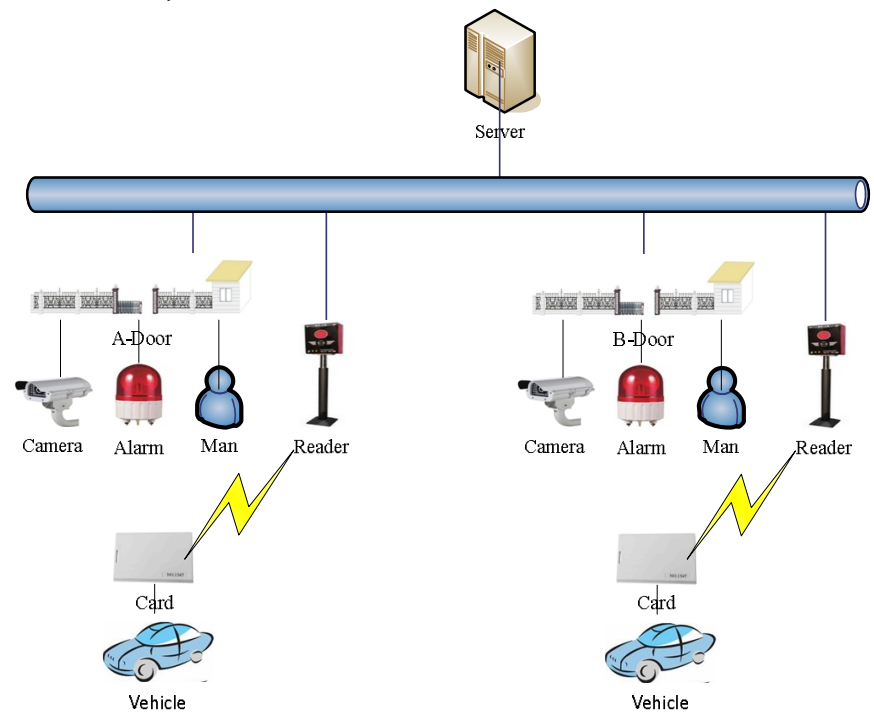

Figure 2 System network structure diagram

\section{Intelligent detection algorithm design}

The intelligent detection algorithm is driven by the self signal of the reader and the RFID card.. The card reader works in real-time scanning card signals, and scans the scanned cards. When a host is connected to a multi card reader, the system first builds a resource pool for each card reader, for saving the card reader read RFID card reader in each desk. 
According to the actual into two scenarios:

i. Scene one: first to identify the car card

1) when the identification card to the car, the system will within a certain interval of time (30 seconds), every 1 second scanning a car card user is bound card:

2) When not in the user to scan the card, alarm processing, alarm and record in the table;

3) When an identification to the user card, to release the vehicle through, and recorded in the import log table.

ii. Scene two: first - to the user identification card

1) when the identification card to the user, the system will in a certain interval of time (such as 10 seconds), every 1 second scanning time and user card bound car card:

2) When all were not scanned car card, the system does not make a deal;

3) In to the car when the identification card, through the release of the vehicle, and recorded in the import log table.

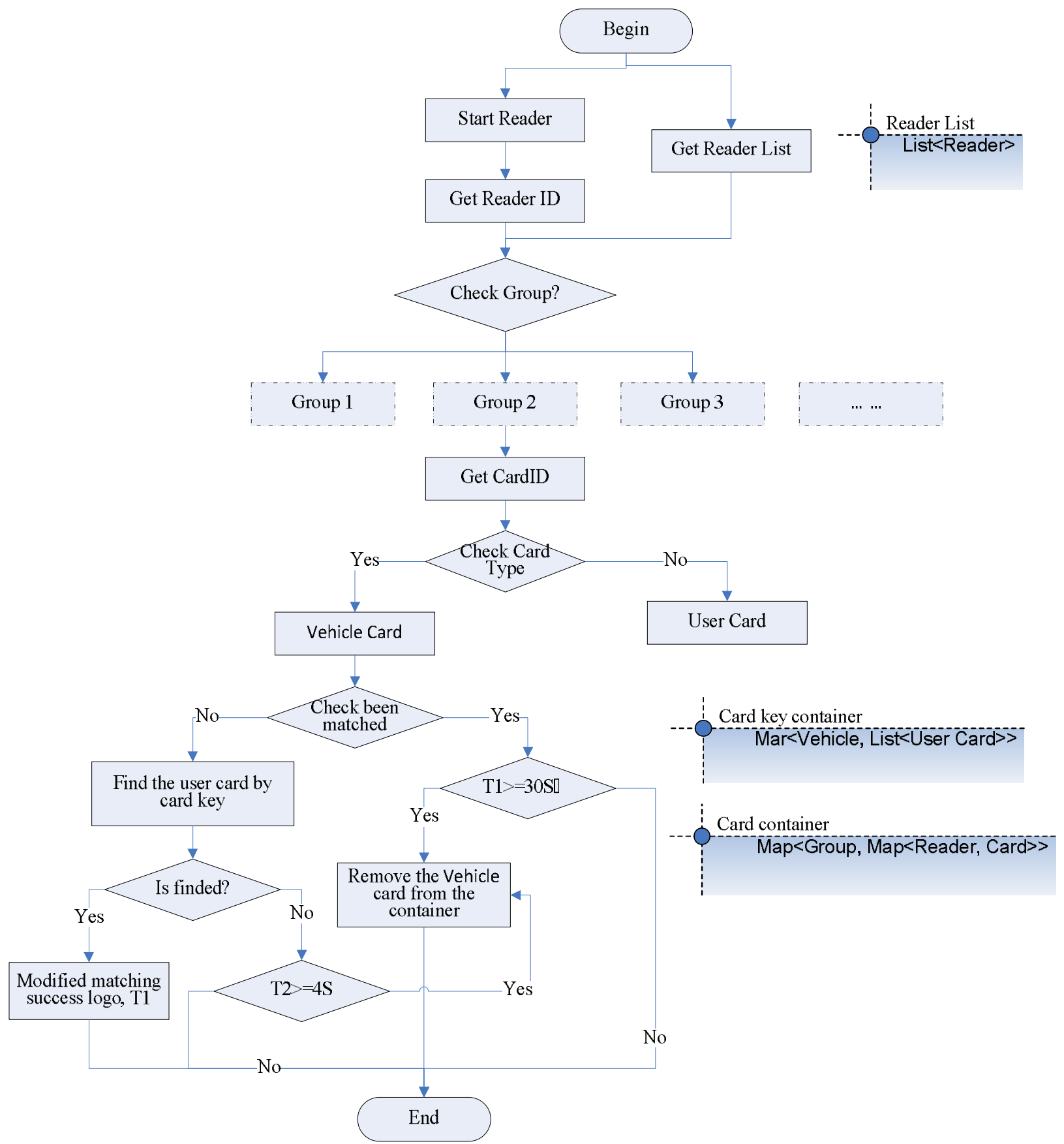

Figure 3 Intelligent detection algorithm flow chart 
The card reader in the boot state after, has been in a state of scanning, when the two scenes, the system will timing time points in the first scan the card, in the time interval to compared to scan all the cards.

The logic of the matching is:

1) When the same group identifies a new card, the user card will be identified within the group matching. If matched, jump to step 2). If the match is not successful, jump to step 3 ..

2) Record of successful identification.

3) The next scan and determine whether a new scan to the car card.

4) If no new car card, then jump to step 3).

5) If there is a new card, then jump to step 1).

6) If scanned to the old car card, then jump to step 3).

7) If the old car card is not scanned, then delete the old car card and jump to step 3.

\section{Conclusion}

At present, RFID technology has been applied in many industries and fields, and has achieved good results.. Through the analysis of the existing problems and current situation of the vehicle security, the intelligent detection and theft system of the vehicle is designed by combining the advantages of RFID technology remote collection.. Can provide the maximum security for people to use the vehicle safety trip, effectively protect the people's property from the loss.

\section{References}

[1] Gao Jianliang, he Jianbiao. RFID principle and Technology (the first set of system networking engineering materials [M]. Electronics Industry Press, 2013.

[2] Li Xinyu, Ceng Chunhui, Pu Limeng. Campus [J]. automatic vehicle management system based on RFID communication technology, 2012,11 (45).

[3] Liang Xingjian, Chen Chao, Qiu Ling. Design and implementation of the archives management system based on RFID [J]. Journal of Sichuan University of Science and Engineering, 2011,6 (24).

[4] Xie Gang, Li Yun, et al. Research on the RFID adaptive collision prevention algorithm based on packet strategy [J]. (23).

[5] Liu Yali, Hu Shengbo, Yan Fuyu. Embedded RFID system recognition rate factor analysis [J]. Journal of engineering design, 2012,06.

[6] Gong Shixiong. Analysis of RFID technology and its application [J]. China scientific and technological information, 2013/03.

[7] Zhang Jiguo, Xu Rui, Wang Dezheng. Study on Jiaxiaotong wireless attendance system [J]. research based on RFID technology, 2011 (12).

[8] Ning Huansheng. RFID network with national major project material (Third Edition) [M]. Machinery Industry Press, 2012

[9] Zhou ye, Yu Xiaosong. The RFID system and the safety problems of [J]. Computer knowledge and technology, 2012 (31).

[10] Yu Jian, Yang Wei. [J]. science and technology and engineering, 2011 (32) based on the direction of active RFID movement. 